\title{
Investigation of Listeria Phage Endolysin Cell-wall Binding Domain (CBD) Surface Display in Escherichia coli
}

\author{
Shan-Na LIU ${ }^{1,2}$, Timo M. TAKALA ${ }^{2}$, Justus REUNANEN ${ }^{3}$, Ossian \\ SARIS ${ }^{2}$ and Per E. J. SARIS ${ }^{2, a,{ }^{*}}$ \\ ${ }^{1}$ College of Food Science and Bioengineering, Tianjin Agricultural University, 22 \\ Jinjing Road, Tianjin 300384, PR China \\ ${ }^{2}$ Department of Food and Environmental Sciences, University of Helsinki, P.O. Box 56, \\ FI-00014 Helsinki, Finland \\ ${ }^{3}$ Department of Veterinary Biosciences, University of Helsinki, P.O. Box 66, FI-00014 \\ Helsinki, Finland \\ aemail: per.saris@helsinki.fi \\ ${ }^{*}$ Corresponding author
}

Keywords: Listeria phage, Endolysin, Cell-wall binding domain (CBD), Surface display.

\begin{abstract}
Cell surface display of target proteins has been widely used in biotechnology and industry. In this study, cell-wall binding domain (CBD) from Listeria phage endolysin A500 was fused with anchoring domains of YadA and OmpA, respectively, in Escherichia coli, aiming at binding of E. coli cells to Listeria cells. The fusion proteins were expressed after induction and their surface localizations were verified by Western blot. CBD-YadA fusion was displayed on the cell surface, however, was toxic to E. coli. OmpA-CBD fusion was translocated to the outer layer of the cell membrane but with compromised availability on the cell surface. Therefore, functional surface display of CBD in $E$. coli requires another anchor for fusion strategy.
\end{abstract}

\section{Introduction}

Listeria monocytogenes is a widely spread foodborne pathogen causing listeriosis with mortality rate of $30 \%$. Contaminated foods contain dairy products, minimally processed vegetables, and ready-to-eat meat products. Listeria phages have been demonstrated as promising candidates in detection, differentiation and biocontrol of Listeria during food processing and storage. Constructed phages with reporter genes such as $\operatorname{lux} A B$ and $c e l B$ have been tested with high sensitivity $[1,2]$. Especially the approval of several Listeria phage commercial agents for food safety purpose promotes research in phage application [3]. Endolysins, the potential candidates for antimicrobials, are bacteriophage-encoded murein hydrolases which can degrade host cell and release phage progeny. The modular structure of Listeria phage endolysins consists of two distinct parts: N-terminal enzymatically active domain (EAD) and C-terminal cell-wall binding domain (CBD) [4]. The EAD part determines the catalytic activity and the CBD part has high binding specificity to Listeria cell wall.

Cell surface display is a rapidly expanded technique in molecular biology and used in whole-cell bioremediation, high-throughput screening and vaccine development. In Gram-negative bacteria, different strategies for displaying proteins have been explored by using carrier proteins such as OmpA, LamB and OprF [5]. Lpp-OmpA is composed of the signal sequence, first nine $\mathrm{N}$-terminal amino acids of $E$. coli lipoprotein (Lpp) 
and 46-159 amino acids of the OmpA. Proteins for instance, hemagglutinin and cyclodextrin glucanotransferase with size ranging from 27 to 74 amino acids have been expressed in this way for biocatalysis and antibody library screening [6]. YadA, originated from Yersinia, is a non-fimbrial adhesin mediating adherence to hosts. Constructed chimeric YadA proteins can translocate the YadA passenger domain across the outer membrane in Yersinia enterocolitica, which provides a clue for potential utilization of YadA anchor domain in cell-surface display [7].

In this study, we aimed at constructing an E. coli strain which would have the ability to bind to pathogenic L. monocytogenes and kill pathogens by introducing anti-listerial peptide (such as bacteriocin) gene. To achieve it, we constructed L. monocytogenes phage endolysins CBD domain surface display systems using anchoring domain YadA and Lpp-OmpA, respectively. The fusion proteins were tested for their expression, location and display on the cell surface.

\section{Materials and Methods}

\section{Bacteria, Plasmids and Culture Conditions}

The E. coli strains and plasmids used in this study are listed in Table 1. E. coli host strains were cultured on Luria-Bertani (LB) medium at $37^{\circ} \mathrm{C}$. Kanamycin $(50 \mu \mathrm{g} / \mathrm{ml})$ and ampicillin $(100 \mu \mathrm{g} / \mathrm{ml})$ were added when appropriate. The expression of CBD-YadA was induced with anhydrotetracycline (1-200 ng/ml), and the expression of OmpA-CBD was induced with $0.02 \mathrm{mM}$ isopropyl- $\beta$-D-thiogalactopyranoside (IPTG). L. monocytogenes WSLC 1018 (gift from Prof. Martin Loessner, ETH Zürich, Switzerland) was cultivated at $30{ }^{\circ} \mathrm{C}$ in BHI medium (Oxoid).

Table 1. E. coli strains and plasmids

\begin{tabular}{|c|c|}
\hline Strains and plasmids & Description \\
\hline \multicolumn{2}{|l|}{ E. coli strains } \\
\hline DH5 $\alpha$ & Transformation host [8] \\
\hline BL21(DE3) & Transformation host (Invitrogen) \\
\hline TG1 & Transformation host [9] \\
\hline ECO615 & TG1/pLEB588 [10] \\
\hline ECO759 & TG1/pLEB733 \\
\hline ECO760 & TG1/pASG-IBA4 \\
\hline ECO762 & TG1/pLEB735 \\
\hline ECO766 & BL21(DE3)/pET-LOAvi \\
\hline ECO767 & BL21(DE3)/pLEB737 \\
\hline \multicolumn{2}{|l|}{ Plasmids } \\
\hline pHPL500 & Vector containing $C B D 500$ gene [11] \\
\hline pET-LOAvi & Cell surface display vector containing lpp-ompA sequence [12] \\
\hline pASG-IBA4 & Vector for CBD-YadA surface display (IBA GmbH) \\
\hline pLEB588 & pHELIX2 + pepR promoter of Lactobacillus rhamnosus [10] \\
\hline pLEB733 & $S S_{u s p 45}-$ papA in pLEB588 \\
\hline pLEB735 & $C B D-y a d A$ in pASG-IBA4 \\
\hline pLEB736 & $\mathrm{P}_{p e p R^{-}}-\mathrm{SS}_{u s p 45^{-}}$papA in pLEB735 \\
\hline pLEB737 & $\begin{array}{l}\text { Vector for OmpA-CBD surface display, derivative of } \\
\text { pET-LOAvi }\end{array}$ \\
\hline
\end{tabular}




\section{Construction of CBD500 Plasmids}

CBD500-YadA fusion was constructed by overlap-PCR with primers CBD500F, CBD500R, yadAF and yadAR (all primer sequences are listed in Table 2). Pediocin gene papA from Lactobacillus plantarum WHE 92 was amplified by PCR with primers PedF and PedR. The signal sequence of $u s p 45$ was amplified by PCR with primers uspF and uspR. The $S S_{u s p 45}$ and papA fragments were cut with NaeI and DraI, ligated, and the ligation mixture was used as a template to amplify $S S_{\text {usp 45-papA fusion with primers }}$

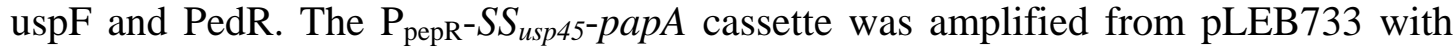
primers pepRF and PedR2. Primers CBD500F2 and CBD500R2 were used to construct OmpA-CBD500 fusion. Plasmids, verified by restriction enzyme digestion and DNA sequencing, was introduced into E. coli BL21(DE3).

Table 2. Primers used in this study

\begin{tabular}{|c|c|}
\hline Name & Sequence ( ${ }^{\prime}$ ' to 3') $^{\prime}$ \\
\hline CBD500F & ACGCCATGGCAAAACACTAATACAAAT \\
\hline CBD500R & ACGCCATGGCAAAACACT AATACAAAT \\
\hline yadAF & ACGCCATGGCAAAACACTAATACAAAT \\
\hline yadAR & ACGCCATGGCAAAACACTAATACAAAT \\
\hline PedF & GAGCTCGAGTTTAAATACTACGGTAATGGGGTTAC \\
\hline PedR & GACGTCGACTAGCATTTATGATTACCT \\
\hline PedR2 & AGTCCACTACGTGCTAGCATTTATGATTACCT \\
\hline uspF & GACGTCGACATGAAAAAAAAGATTATCTCAGC \\
\hline uspR & GAGCTCGAGGCCGGCGTAAACACCTGACAACGG \\
\hline pepRF & ACTGCACATTGTGTGCTTTGATACTCACCAATG \\
\hline CBD500F2 & ACGCCATGGCAAAACACTAATACAAAT \\
\hline CBD500R2 & AACGGATCCTTAGTGATGGTGATGGTGATGTTTTAAGAAGTATTC \\
\hline
\end{tabular}

\section{Cell Fractionation}

Bacterial cell fractionation was carried out as previously described [13]. Cell suspension was then sonicated on ice. The supernatant was centrifuged at $25,000 \times \mathrm{g}$ for $1 \mathrm{~h}$ to obtain total membrane fraction in pellet. The supernatant was regarded as the soluble cytoplasmic/periplasmic fraction. The pellet was resuspended with $20 \mathrm{mM}$ Tris- $\mathrm{HCl}$ buffer (containing 1\% sodium lauroyl sarcosine, $\mathrm{pH}$ 7.4) for solubilizing inner membrane. The outer membrane fraction was re-pelleted by centrifugation at $25,000 \times \mathrm{g}$ at $4{ }^{\circ} \mathrm{C}$ for $1 \mathrm{~h}$. The supernatant was regarded as the inner membrane fraction.

\section{SDS-PAGE and Western Blot Analysis}

The protein samples were analysed on $12 \%$ SDS-PAGE. Separated proteins were electroblotted onto polyvinylidene-fluoride (PVDF) membranes $(0.45 \mu \mathrm{m})$ (Millipore) in transfer buffer (25 mM Tris, $192 \mathrm{mM}$ glycine, 20\% methanol). The anti-His/AP antibody was used for Western blot analysis (Invitrogen WesternBreeze chromogenic kit) according to manufacturer's instructions.

\section{Whole Cell ELISA}

Induced cells of pASG-IBA4 or pET-LOAvi constructs were harvested, washed with PBS ( $\mathrm{pH}$ 7.0), and resuspended in the same buffer to an OD600 $=1$. Cells were coated 
onto the wells of 96 wells Nunc microtiter plate overnight at $4{ }^{\circ} \mathrm{C}$ and blocked with $3 \%$ BSA in PBS. The wells were washed with PBST (PBS and $0.1 \%$ Tween 20). The pET-LOAvi constructs were incubated with anti-His/AP antibody at a final dilution of 1:3000 for $1 \mathrm{~h}$. Wells were washed with PBST before adding alkaline phosphatase yellow (pNPP) as a substrate. The absorbance of each well was measured at $405 \mathrm{~nm}$. For pASG-IBA4 constructs, anti-Strep-tag antibody (1:500), anti-rabbit antibody with horseradish peroxidase conjugate (1:5000), and TMB as a substrate were used. After stopping the color reaction with $0.5 \mathrm{M} \mathrm{H}_{2} \mathrm{SO}_{4}$, the absorbance was measured at $450 \mathrm{~nm}$.

\section{Results}

\section{Surface Expression of CBD500 with the Outer Membrane Anchor of YadA}

CBD500 (GenBank: X85009.1), originated from L. monocytogenes phage endolysin Ply500 has specificity to bind to the surface of Listeria cells [4]. By whole cell ELISA the CBD-YadA fusion was shown to be displayed on the cell surface in strain ECO762 in Fig. 1A. Addition of anti-listerial peptide pediocin secretion to ECO762 cells decreased the production of the CBD500-YadA fusion protein (Fig. 1). Furthermore, tetracycline induction of CBD500-YadA expression inhibited cell growth and induced cell lysis (data not shown). Since the CBD500-YadA fusion was toxic for E. coli, and the co-production of pediocin further weakened its expression, the next aim was to test another membrane anchor for the surface display of CBD500.

A

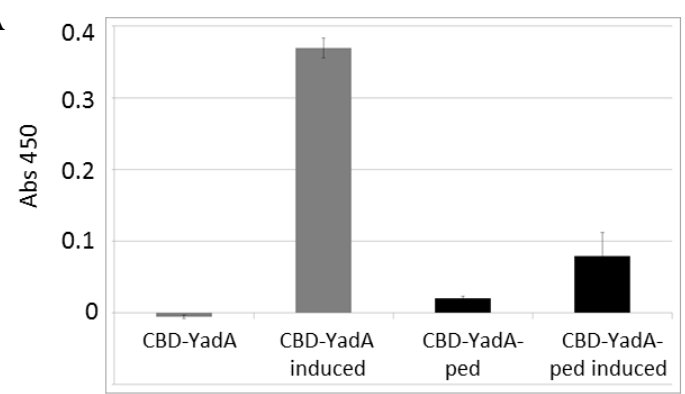

B

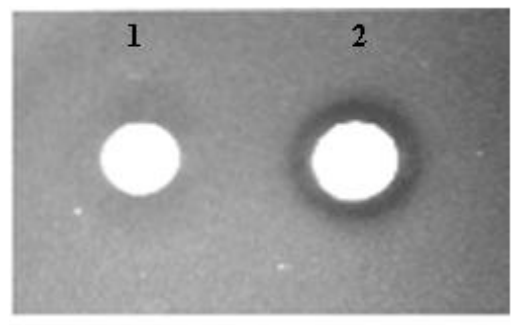

Figure 1. Analysis of E. coli YadA constructs. A: Whole cell ELISA of CBD500-YadA and CBD500-YadA-pediocin (ped) fusions expressed on the surface of tetracycline-induced ECO762 (TG1/CBD500-YadA) and ECO764 (TG1/CBD500-YadA + $\mathrm{P}_{p e p R^{-}}-S_{\text {usp } 45-p a p A)}$ cells respectively. Co-production of pediocin reduces the CBD500-YadA expression. B: Inhibition of L. monocytogenes WSLC 1018 by pediocin producing E. coli. 1, ECO762 cells; 2, ECO764 cells. Two $\mu 1$ of the culture suspensions were spotted on Listeria lawn on BHI agar plate, and incubated overnight.

\section{Availability of the OmpA-CBD500 Fusion Protein on the Cell Envelope}

OmpA-CBD500 was expressed at reasonable level after IPTG induction. Fig. 2A shows a visible new protein band estimated to be around $30 \mathrm{kDa}$ could be detected as expected by SDS-PAGE. The localization of the CBD500 on the external surface of $E$. coli was determined by Western blot. Strong bands could be observed in the outer membrane fraction after IPTG induction, indicating the CBD500 fusion was mainly localized at the outer layer of the cell membrane (Fig. 2A). The availability of the CBD500 domain on the surface of intact cells was analysed by whole cell ELISA. The signal of ELISA was weak, though higher than the negative control (Fig. 2B). Displaying CBD500 via OmpA anchor did not show evident binding effect when mixing induced $E$. coli cells with listerial cells (data not shown). 
A

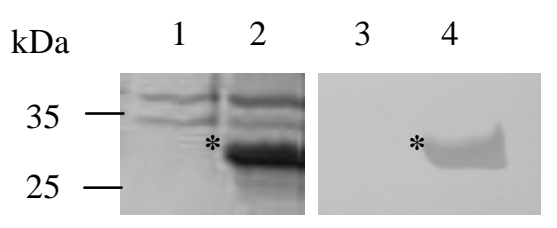

B

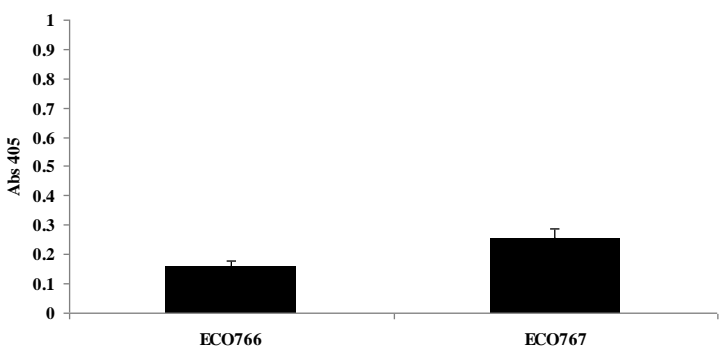

Figure 2. Analysis of E. coli OmpA constructs. A: Localization of OmpA-CBD500. 1, SDS-PAGE of outer membrane proteins without IPTG induction; 2, SDS-PAGE of outer membrane proteins with IPTG induction; 3, Western blot of outer membrane proteins without IPTG induction; 4, Western blot of outer membrane proteins with IPTG induction. The OmpA-CBD500 fusion protein is marked with an asterisk. B: Whole cell ELISA of ECO766 (BL21(DE3)/pET-LOAvi) and ECO767(BL21(DE3)/OmpA-CBD500) after IPTG induction.

\section{Discussion}

Previously, Listeria phage endolysins CBDs have been fused with green fluorescence protein (gfp) to study their binding mechanisms with listerial cells [4]. However, there is no report about presenting CBDs on the microbial surface via outer membrane protein fusions and investigating the interaction by in vivo model. An evident outcome is surface display of anchor YadA is not favorable but lethality in E. coli cells. Expression of CBD500 fused to OmpA was successful, but the protein on the surface was poorly available for antibodies. It is speculated the presented CBD500 is not far enough away from the lipopolysaccharide layer, which may prevent the interaction between CBD500 and Listeria cell wall ligands. Or, the CBD500 has penetrated into outer membrane after translocation, leading to few accessible CBD500 for binding. Another concern is the putative incorrect folding of CBD500 due to high level expression under T7 promoter. Stathopoulos et al [14] have pointed out earlier this display system is probably not compatible to extensive secondary and tertiary structures of the passenger protein. As the three dimensional structure of CBD500 and the specific region responsible for binding have not been revealed, one can speculate conformation change may have happened in the fusion potentially explaining the inefficient interactions with ligands.

\section{Conclusions}

CBD500 displaying E. coli strains were constructed using YadA and OmpA as carrier proteins. Fusion proteins were expressed on the cell surface with compromised availability on the cell envelope. Utilization of outer membrane protein anchors for surface display may require introduction of long proteins linkers or protein domains to ensure functional presentation of the domain to be displayed.

\section{Acknowledgement}

This work was supported by the University of Helsinki, Finland, the Academy of Finland (project number 177321), the Key Technology R \& D Program of Tianjin (14ZCZDNC00003) and the National Natural Science Foundation of China (Project 31501583). Professor Martin Loessner is acknowledged for the kind gifts of the Listeria strain and the CBD500 encoding DNA. 


\section{References}

[1] M. J. Loessner, M. Rudolf, S. Scherer, Appl. Environ. Microb. 63 (1997) 2961-2965.

[2] S. Hagens, T. de Wouters, P. Vollenweider, M. J. Loessner, Bacteriophage 1 (2011) 143-151.

[3] J. Mahony, O. McAuliffe, R.P. Ross, D. van Sinderen, Curr. Opin. Biotech. 22 (2011) 157-163.

[4] M. J. Loessner, K. Kramer, F. Ebel, S. Scherer, Mol. Microbiol. 44 (2002) 335-349.

[5] P. Samuelson, E. Gunneriusson, P-Å. Nygren, S. Ståhl, J. Biotechnol. 96 (2002) 129-154.

[6] E. Bloois, R. T. Winter, H. Kolmar, M. W. Fraajie, Trends Biotechnol. 29 (2011) $79-86$

[7] N. Ackermann, M. Tiller, G. Anding, A. Roggenkamp, J. Heesemann, J. Bacteriol. 190 (2008) 5031-5043.

[8] D. Hanahan, J. Mol. Biol. 166 (1983) 557-580.

[9] T. J. Gibson, Studies on the Epstein-Barr virus genome, $\mathrm{PhD}$ thesis, University of Cambridge, UK, 1984.

[10] T. M. Takala, P. E. Saris, S. S. Tynkkynen, Appl. Microbiol. Biot. 60 (2003) 564-570.

[11] M. J. Loessner, A. Schneider, S. Scherer, Appl. Environ. Microb. 62 (1996) 3057-3060.

[12] Z. Yang, Q. Liu, Q. Wang, Y. Zhang, Appl. Environ. Microb. 74 (2008) 4359-4365.

[13] J. Y. Wang, Y. P. Chao, Appl. Environ. Microb. 72 (2006) 927-931.

[14] C. Stathopoulos, G. Georgiou, C. F. Earhart, Appl. Microbiol. Biot. 45 (1996) 112-119. 